\title{
Phonon-induced polariton superlattices
}

de Lima, Jr., M. M.; Poel, Mike van der; Santos, P. V.; Hvam, Jørn Märcher

Published in:

Physical Review Letters

Link to article, DOI:

10.1103/PhysRevLett.97.045501

Publication date:

2006

Document Version

Publisher's PDF, also known as Version of record

Link back to DTU Orbit

Citation (APA):

de Lima, Jr., M. M., Poel, M. V. D., Santos, P. V., \& Hvam, J. M. (2006). Phonon-induced polariton superlattices. Physical Review Letters, 97(4), 045501. https://doi.org/10.1103/PhysRevLett.97.045501

\section{General rights}

Copyright and moral rights for the publications made accessible in the public portal are retained by the authors and/or other copyright owners and it is a condition of accessing publications that users recognise and abide by the legal requirements associated with these rights.

- Users may download and print one copy of any publication from the public portal for the purpose of private study or research.

- You may not further distribute the material or use it for any profit-making activity or commercial gain

- You may freely distribute the URL identifying the publication in the public portal

If you believe that this document breaches copyright please contact us providing details, and we will remove access to the work immediately and investigate your claim. 


\title{
Phonon-Induced Polariton Superlattices
}

\author{
M. M. de Lima, Jr., ${ }^{1, *}$ M. van der Poel, ${ }^{2}$ P. V. Santos, ${ }^{1}$ and J. M. Hvam ${ }^{2}$ \\ ${ }^{1}$ Paul-Drude-Institut für Festkörperelektronik, Hausvogteiplatz. 5-7, 10117 Berlin, Germany \\ ${ }^{2} C O M \bullet D T U$, NanoDTU, Technical University of Denmark, DK-2800 Kongens Lyngby, Denmark \\ (Received 15 February 2006; published 24 July 2006)
}

\begin{abstract}
We show that the coherent interaction between microcavity polaritons and externally stimulated acoustic phonons forms a tunable polariton superlattice with a folded energy dispersion determined by the phonon population and wavelength. Under high phonon concentration, the strong confinement of the optical and excitonic polariton components in the phonon potential creates weakly coupled polariton wires with a virtually flat energy dispersion.
\end{abstract}

DOI: 10.1103/PhysRevLett.97.045501

The coherent control of elementary excitations in condensed matter through spatial confinement effects is a major research topic. In recent years, methods have been sought for the control of dimensionality in a dynamic way using external fields. The dimensionality control normally requires fields with a spatial variation significantly smaller than the phase coherence length of the elementary excitation. This constraint can be generally satisfied for weakly interacting particles, such as photons or low-frequency phonons [1]. The stronger interaction of electronic excitations with their environment, in contrast, substantially reduces their coherence length, thus requiring field variations in the nanometer range to induce dimensionality effects.

The phase coherence length of electronic excitations can be significantly enhanced by mixing them with photons. An outstanding example is the microcavity (MC) polariton-a quasiparticle formed through the strong coupling of excitons with photons in a semiconductor MC [2]. In the polaritons, the photons act as a clock that synchronizes excitonic resonances, thus imparting a longer time and spatial coherence to polariton states as compared to bare excitons [3]. The enhanced coherence has motivated basic investigations of micro-optical parametric oscillators [4] as well as ultrafast optical amplifiers [5]. Successful tuning of MC polaritons, without changing their dimensionality, has been demonstrated using static electric [6] and strain fields [7]. Conversely, the static reduction of polariton dimensionality using lithographically defined structuring has also been reported [8-11]. Tignon et al. [12] have shown the dynamic reduction in dimensionality of polariton states through the formation of Landau levels under a magnetic field. Recently, Ivanov and Littlewood have proposed the use of acoustic fields for the coherent modulation of MC polaritons through an acoustically induced Stark effect [13]. They argue that the strong exciton-photon coupling inherent to the polaritons significantly enhances the light scattering cross section by acoustic phonons with important consequences for acousto-optic devices.

In this Letter, we demonstrate the dynamic control of dimensionality of MC polaritons through the coherent interaction with acoustic phonons in the form of
PACS numbers: 63.20.Ls, 42.50.-p, 71.35.-y, 73.21.Cd

Rayleigh surface acoustic waves (SAWs). Using a comprehensive theoretical model, we show that the periodic acoustic field modulates both the optical and electronic polariton components along the phonon propagation direction $(\hat{x}$, cf. Fig. 1), resulting in a dynamic polariton superlattice with a period equal to the phonon wavelength $\left(\lambda_{\mathrm{SAW}}\right)$. The dynamic character of the modulation allows us to follow the transition from a two-dimensional to a onedimensional polariton dispersion as the confinement potential increases with the phonon population. For low population, one observes additional reflectivity resonances associated with the folded dispersion modes. As the population increases, energy gaps open up in the dispersion due to the interaction between these modes. The transition to one-dimensional behavior takes place when the amplitude of the phonon-induced energy modulation exceeds the energetic width of the dispersion branch. Due to the weak energy dispersion, this effect is more pronounced for the lowest (or fundamental) dispersion branch, leading to the formation of an array of identical weakly coupled polariton wires oriented along $\hat{y}$. Diffraction experiments demonstrate that these wires reemit coherently under opti-

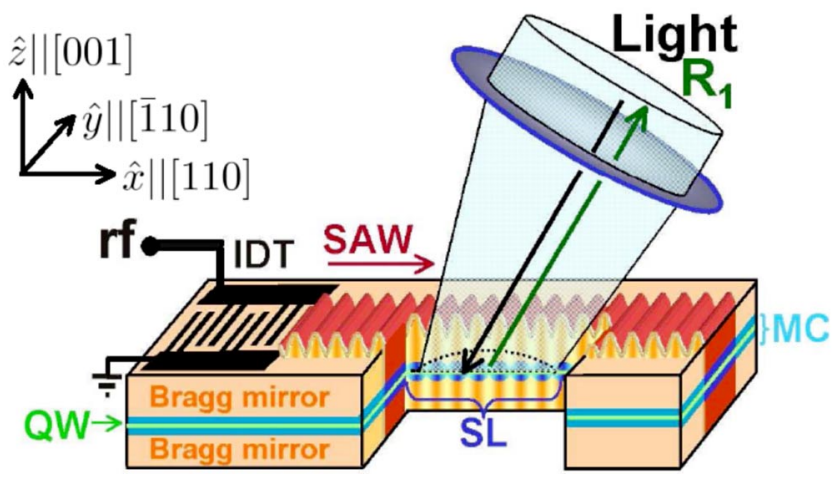

FIG. 1 (color online). Schematic diagram of a (Al, Ga)As MC with an embedded QW. The structure is modulated by a SAW excited by an IDT forming a polariton superlattice (SL). Also shown is the geometry for diffraction experiments, where one detects the first-order diffraction (with intensity $R_{1}$ ) of a light beam impinging at the Bragg angle. 
cal excitation, thus opening the way for new concepts in quantum optics as well as optoelectronic applications.

The studies were performed using an (Al, Ga)As sample grown by molecular-beam epitaxy with the structure sketched in Fig. 1 [14], on which a SAW with $\lambda_{\mathrm{SAW}}=$ $5.6 \mu \mathrm{m}$ is electrically stimulated by an interdigitated transducer (IDT). The GaAs spacer layer containing the quantum wells (QWs) is wedged in order to yield MC polaritons with different detuning energies $\delta=\hbar\left(\omega_{\ell}-\omega_{\chi}\right)$ between the uncoupled photonic $\left(\hbar \omega_{\ell}\right)$ and excitonic $\left(\hbar \omega_{\chi}\right)$ resonant energies along $\hat{x}$. The vacuum Rabi splitting was measured to be $4.0 \mathrm{meV}$.

We start with a theoretical description of the coherent interaction between MC polaritons and phonons. In the absence of acoustic pumping, the dispersion of uncoupled photons (subscript $\rho=\ell$ ) and excitons $(\rho=\chi)$ are given by $\hbar \omega_{\rho}\left(k_{x}\right) \approx \hbar \omega_{\rho_{0}}+\hbar^{2} k_{x}^{2} /\left(2 m_{\rho}\right)$, where $k_{x}$ is the inplane wave vector, $\hbar \omega_{\rho 0}$ denotes the energy of the excitation for $k_{x}=0$, and $m_{\rho}$ is the effective particle mass [ $m_{\ell}=$ $\left.\pi n_{c} /\left(c d_{c}\right)\right]$. The SAW strain field simultaneously changes the thickness $\left(d_{c}\right)$ and the refractive index $\left(n_{c}\right)$ of the spacer layer. The SAW strain field in the cavity region consists of three components $\varepsilon_{i j}(z, t)=\operatorname{Re}\left[\tilde{\varepsilon}_{i j}(z) e^{i \varphi \mathrm{SAW}}\right]$, $i j=x x, z z, x z$. Here $k_{\mathrm{SAW}}=2 \pi / \lambda_{\mathrm{SAW}}$, and $\varphi_{\mathrm{SAW}}=$ $k_{\mathrm{SAW}} x-\omega_{\mathrm{SAW}} t$ is the SAW phase. The complex amplitudes $\tilde{\varepsilon}_{i j}$ were determined using the elastic model for SAW propagation described in Refs. $[15,16]$. Using these results, the spatial modulation of $\omega_{\ell 0}$ becomes $[1,16]$

$$
\frac{\Delta \tilde{\omega}_{\ell}}{\omega_{\ell_{0}}}=-\tilde{\varepsilon}_{z z}-\frac{n_{c}^{2}}{2}\left[\left(p_{11}+p_{12}+2 p_{44}\right) \frac{\tilde{\varepsilon}_{x x}}{2}+p_{12} \tilde{\varepsilon}_{z z}\right] .
$$

The first term on the right-hand side represents the thickness modulation while the elasto-optic contribution (second term) is described in terms of the elasto-optic tensor $p_{i j}$ of the medium and applies for light polarized along the [110] direction $(\hat{y})$. In addition, the strain modulates the excitonic energy $\hbar \omega_{\chi}$ through the deformation potential (DP) interaction according to

$$
\frac{\Delta \tilde{\omega}_{\chi}}{\omega_{\chi_{0}}}=\left[a_{h}\left(\tilde{\varepsilon}_{x x}+\tilde{\varepsilon}_{z z}\right)+b\left(\tilde{\varepsilon}_{z z}-\frac{\tilde{\varepsilon}_{x x}}{2}\right)\right],
$$

where $a_{h}$ and $b$ are the hydrostatic and the valence band uniaxial DPs, respectively [17-19]. Figure 2(a) compares the spatial modulation of the photonic and excitonic resonances in the MC structure under a SAW with a linear power density $P_{\ell}=100 \mathrm{~W} / \mathrm{m}$ (defined as the acoustic power per unit length perpendicular to the propagation direction). Note that $\Delta \omega_{\chi}>2 \Delta \omega_{\ell}$ as a result of the stronger coupling of phonons to excitons [20].

The periodic perturbations expressed by Eqs. (1) and (2) fold the polariton dispersion along $\hat{x}$ within a miniBrillouin zone (MBZ) of extent $k_{\mathrm{SAW}}$ by coupling states with wave vectors differing by a multiple of $k_{\mathrm{SAW}} \hat{x}$. The upper panels in Fig. 3 show the first four branches of the

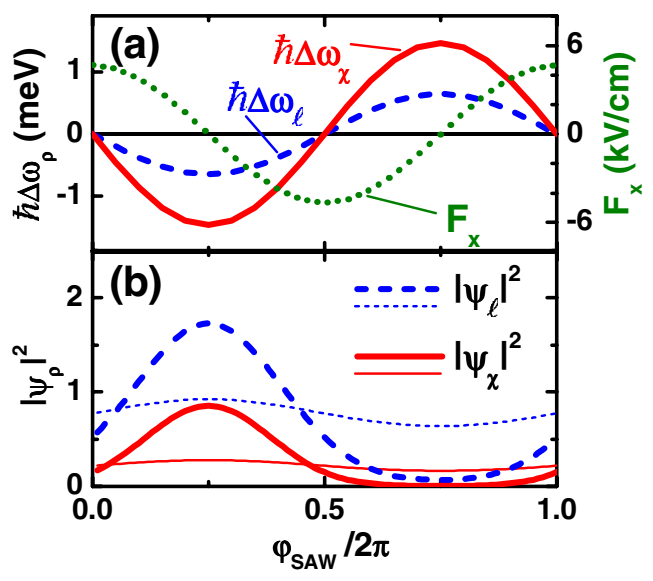

FIG. 2 (color online). Dependence of (a) the photonic $\left(\hbar \omega_{\ell}\right)$ and excitonic energies $\left(\hbar \omega_{\chi}\right)$ and (b) corresponding wave functions for the first zone-center mode for $\delta=-2.7 \mathrm{meV}$ on the SAW phase $\left(\varphi_{\mathrm{SAW}}=k_{\mathrm{SAW}} x-\omega_{\mathrm{SAW}} t\right)$, calculated for an acoustic mode with $P_{\ell}=100 \mathrm{~W} / \mathrm{m}$ and $\lambda_{\text {SAW }}=5.6 \mu \mathrm{m}$. The dotted line in (a) shows the longitudinal piezoelectric field $F_{x}$ induced by the SAW. The thin lines in (b) display, for comparison, the wave functions calculated for a low power $\left(P_{\ell}=1 \mathrm{~W} / \mathrm{m}\right)$.

lower (LP) and upper (UP) polariton dispersion curves calculated for three different detunings $\delta$ using the procedure described in Ref. [16]. The folded dispersion presents gaps at its center and boundaries, which increase with the acoustic intensity. The fundamental (i.e., the lowest energy) dispersion branch becomes essentially flat for high acoustic powers, thus indicating the formation of a periodic array of weakly coupled polariton wires oriented along $\hat{y}$. In fact, the envelopes of the photonic $\left(\psi_{\ell}\right)$ and excitonic $\left(\psi_{\chi}\right)$ wave function components, obtained from the calculated polariton eigenstates, become localized, as displayed in Fig. 2(b) for the zone-center mode (i.e., $k_{x}=0$ ). These envelopes, which were calculated for $\delta=-2.7 \mathrm{meV}$, vary little for $0<k_{x}<k_{\mathrm{SAW}} / 2$.

The dispersion of the additional branches is determined by the photon-exciton admixture of the modes. For negative detunings [cf. Figs. 3(a) and 3(b)], the LP branches are predominantly of photonic character. Because of the lower photon effective mass, the dispersion of these branches is much more pronounced than the ones for the UP branches, which are mainly excitonic. The situation is reversed for positive detunings [cf. Fig. 3(c)].

The experimental evidence for folded dispersion modes at the zone center was provided by normal-incidence reflectivity $\left(R_{0}\right)$ measurements at $20 \mathrm{~K}$. A modulation technique was employed to correct for temperature changes in the spectra due to heating at high excitation powers. Figures 3(d)-3(f) show $R_{0}$ measured for the detunings indicated in the respective upper panels. In the absence of acoustic excitation, the reflectivity shows two resonance dips associated with the excitation of the $k_{x}=0$ modes of the LP and UP branches and oscillator strengths reflecting the photonic content. Under acoustic pumping, these 


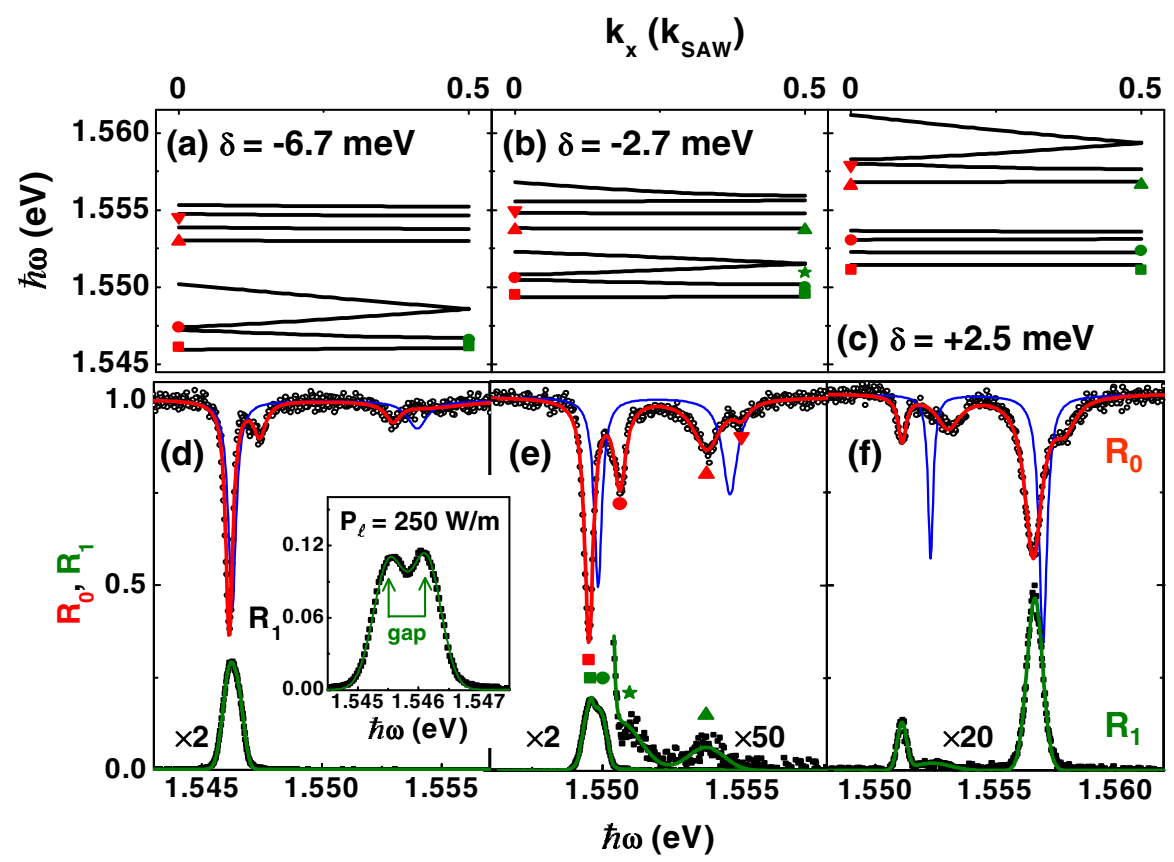

FIG. 3 (color online). Upper panels: (a)-(c) Calculated polariton dispersion for different detunings $\left[\delta=\hbar\left(\omega_{\ell}-\omega_{\chi}\right)\right]$. Lower panels: (d)-(f) Normal-incidence reflectivity $R_{0}$ (open dots, upper plots) and first-order back-diffraction $R_{1}$ (solid dots, lower plots) measured under a SAW for $\delta$ indicated in the respective upper panels. $R_{0}$ spectra in the absence of a SAW (thin lines) are also shown for comparison. All calculations and measurements were performed for an acoustic power $P_{\ell}=100 \mathrm{~W} / \mathrm{m}$ except for the $R_{1}$ spectrum in the inset in (d), which was recorded for $P_{\ell}=250 \mathrm{~W} / \mathrm{m}$. The symbols in the upper panels and in (e) represent the energy positions obtained from the fits (lines superimposed to the experimental data) displayed in the lower panels. The vacuum Rabi splitting used in the calculations corresponds to the measured value of $4.0 \mathrm{meV}$.

modes redshift, and additional resonances appear at higher energies. The latter are attributed to the excitation of folded dispersion modes. This assignment is reinforced by the good agreement between the calculated and measured resonance energies illustrated in Figs. 3(a)-3(c). In addition, the model describes well the dependence of the energies on acoustic power shown in Fig. 4(a). The modes indicated by dashed lines in Fig. 4(a) have, due to their symmetry, a weak oscillator strength and are not observed in the experiments.

Direct evidence for the long-range coherence of the folded polaritons is provided by diffraction experiments using the configuration indicated in Fig. 1 [21]. Here the zone-edge modes (i.e., with $k_{x}=k_{\mathrm{SAW}} / 2$ ) are probed by illuminating the polariton grating at the Bragg angle and recording the backscattered first-order diffraction beam $\left(R_{1}\right)$ [22]. As indicated in the lower panels in Fig. 3, diffraction is observed from both polariton branches. The diffraction lines split into two with increasing acoustic power, as the energy gap between the first two zone-edge states exceeds the spectral linewidths. This behavior is particularly evident in the spectrum recorded for a higher power $\left(P_{\ell}=250 \mathrm{~W} / \mathrm{m}\right)$ shown in the inset in Fig. 3(d). The splitting, which has been previously reported for bare cavities (i.e., without QWs) [1,16], directly demonstrates the formation of an energy gap through the strong interaction between the polariton modes with wave vectors $k_{x}=$ $\pm k_{\mathrm{SAW}} / 2$ mediated by the phonons. The power depen- dence of the energy of the zone-edge resonances is also in very good agreement with the calculations, as shown in Fig. 4(b). Finally, the shoulder indicated by the star in Fig. 3(e) provides an experimental verification of the dif-

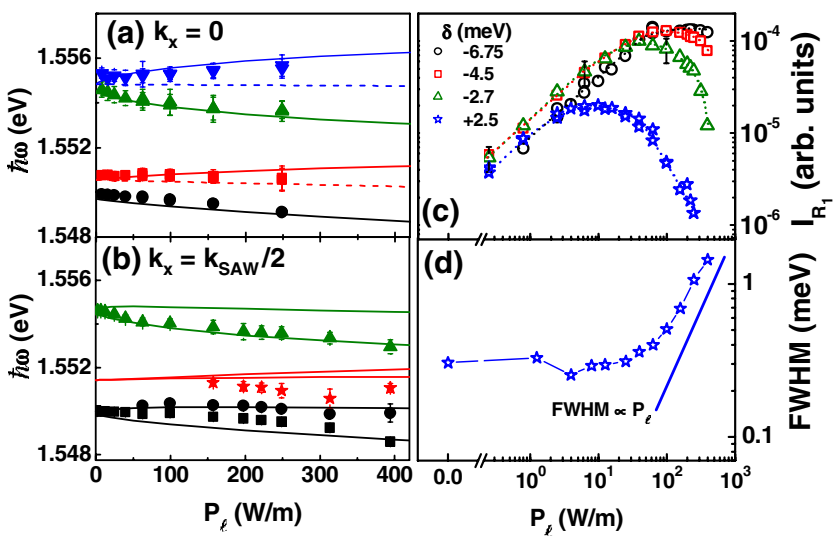

FIG. 4 (color online). Acoustic power density dependence of the (a) zone-center and (b) zone-edge energies for a detuning $\delta=-2.7 \mathrm{meV}$. The lines are the calculated dispersion branches. The dashed lines represent modes with weak oscillator strength. (c) Integrated back-diffraction intensity $\left(I_{R_{1}}\right)$ from the lower polariton branch measured for different detunings $\delta$. The dotted lines are guides to the eyes. (d) Full width at half maximum (FWHM) of the fundamental polariton mode for $\delta=$ $+2.5 \mathrm{meV}$. 
fraction from higher-order folded modes [from the third branch at the edge of the MBZ, cf. Fig. 3(b)] predicted in Ref. [23].

Diffraction is a coherent process: Its integrated intensity $\left(I_{R_{1}}\right)$ depends both on the density and on the phase coherence of the excited modes. The density is determined by the flatness of the dispersion at the band edge, which increases with the modulation amplitude and dominates the behavior of $I_{R_{1}}$ from the LP branch for low acoustic powers and negative detunings [cf. Fig. 4(c)]. Decoherence effects, in contrast, become important under high acoustic excitation. In fact, the diffraction intensity in Fig. 4(c) reaches a maximum at a power density that decreases with increasing excitonic content. This unusual behavior, which differs from the monotonic increase in $I_{R_{1}}$ observed in bare cavities [1], is attributed to a reduction of the polariton phase coherence induced by exciton scattering. The polariton coherence time (on the order of $2 \mathrm{ps}$ in the absence of SAW) shortens as excitons are either ionized or scattered while moving in the potential landscape produced by the acoustic field. The decoherence is also corroborated by the increase of the linewidths with the phonon population ( $\propto P_{\ell}$, for high $P_{\ell}$ ), as illustrated in Fig. 4(d) for the fundamental polariton mode and $\delta=+2.5 \mathrm{meV}$. Note that, in contrast to the interaction with thermal phonons, the excitons here coherently interact with the strain field of a single, well-defined vibrational mode [13]. The longitudinal piezoelectric field $F_{x}$, in contrast, effectively ionizes excitons and reduces radiative recombination by spatially separating electrons and holes [16]. The calculated profiles for $F_{x}$, illustrated by the dotted line in Fig. 2(a), do not preclude the existence of polaritons with long coherence lengths. In fact, $F_{x}$ vanishes close to the maximum of $\left|\psi_{\chi}\right|^{2}$ so that excitons within this region remain essentially unaffected. The spatial extent of this region decreases with increasing $F_{x}$, leading to a lower population of coherent states contributing to diffraction. These decoherence effects have important consequences for optical modulation, since they limit the maximum diffraction amplitudes and can be avoided by exciting superlattices along nonpiezoelectric crystallographic directions.

In conclusion, we have demonstrated the formation of a dynamic polariton superlattice through the modulation of MC polaritons by stimulated acoustic phonons. The spatial modulation of the excitonic and photonic energies creates a folded polariton dispersion with well-defined stop bands. For high phonon concentrations, the modes of the fundamental branch are weakly coupled polariton wires, which reemit coherently under acoustic excitation. Since the dynamic confinement is controlled solely by the phonon population and wavelength, the polariton wires are identical and virtually free of potential fluctuations, in contrast to static ones defined by lithography [8-11]. Note that unavoidable inhomogeneities in the IDTs introduced by the lithographic fabrication, while detrimental to the electroacoustic conversion efficiency, do not add potential fluctu- ations to the wires. In addition, periodic arrays of confined polaritons with different potential shapes can be generated and controlled by interfering SAW beams propagating along different directions [1]. Tunability is achieved by simply controlling the SAW wavelength, amplitude, or relative phase. Finally, the coherent control of essentially identical confined polaritons using acoustic fields demonstrated here opens the way for new concepts in quantum optics that can be exploited to manipulate the polariton density and to enhance nonlinear effects arising from polariton-polariton interactions [10].

We thank A. Cantarero, S. Fölsch, and K. H. Ploog for comments and for a critical reading of the manuscript, as well as J.R. Jensen, S. Krauß, and W. Seidel for the growth and preparation of the samples. Support from the EU Network of Excellence ePIXnet is gratefully acknowledged.

*Electronic address: mmlimajr@pdi-berlin.de

[1] M. M. de Lima, Jr. et al., Phys. Rev. Lett. 94, 126805 (2005).

[2] C. Weisbuch et al., Phys. Rev. Lett. 69, 3314 (1992).

[3] A. I. Tartakovskii et al., Phys. Status Solidi B 221, 163 (2000).

[4] J. J. Baumberg et al., Phys. Rev. B 62, R16 247 (2000).

[5] P. G. Savvidis et al., Phys. Rev. Lett. 84, 1547 (2000).

[6] T. A. Fisher et al., Phys. Rev. B 51, 2600 (1995).

[7] R. B. Balili et al., Appl. Phys. Lett. 88, 031110 (2006).

[8] A. I. Tartakovskii et al., J. Exp. Theor. Phys. 87, 723 (1998).

[9] A. Kuther et al., Phys. Rev. B 58, 15744 (1998).

[10] G. Dasbach et al., Phys. Rev. B 66, 201201(R) (2002).

[11] O. E. Daïf et al., Appl. Phys. Lett. 88, 061105 (2006).

[12] J. Tignon et al., Phys. Rev. Lett. 74, 3967 (1995).

[13] A. L. Ivanov and P. B. Littlewood, Phys. Rev. Lett. 87, 136403 (2001).

[14] J. R. Jensen et al., Appl. Phys. Lett. 76, 3262 (2000).

[15] S. H. Simon, Phys. Rev. B 54, 13878 (1996).

[16] M. M. de Lima, Jr. and P. V. Santos, Rep. Prog. Phys. 68, 1639 (2005).

[17] G. L. Bir and G. E. Pikus, Symmetry and Strain-Induced Effects in Semiconductors (Wiley, New York, 1974).

[18] P. Yu and M. Cardona, Fundamentals of Semiconductors: Physics and Materials Properties (Springer, Heidelberg, 1995).

[19] P. V. Santos et al., Phys. Rev. B 69, 155318 (2004).

[20] A. L. Ivanov and P. B. Littlewood, Semicond. Sci. Technol. 18, S428 (2003).

[21] M. M. de Lima, Jr., R. Hey, and P. V. Santos, Appl. Phys. Lett. 83, 2997 (2003).

[22] The validation of the diffraction experiments as a means of accessing the modes at the edge of the phonon-induced mini-Brillouin zone was already demonstrated for bare cavities (i.e., without QWs) [1,16], where this technique was used to access modes along different directions of a two-dimensional lattice generated by SAW beams.

[23] K. Cho et al., Phys. Rev. Lett. 94, 226406 (2005). 